
centrasiatiques et tibétaines

$45 \mid 2014$

Épopée et millénarisme : transformations et innovations

\title{
L'exaltation des Roerich au Petit Tibet, ou à la naissance du New Age
}

Dany Savelli

\author{
(2) OpenEdition \\ Journals \\ Édition électronique \\ URL : https://journals.openedition.org/emscat/2423 \\ DOI : $10.4000 /$ emscat.2423 \\ ISSN : 2101-0013 \\ Éditeur \\ Centre d'Etudes Mongoles \& Sibériennes / École Pratique des Hautes Études \\ Référence électronique \\ Dany Savelli, "L'exaltation des Roerich au Petit Tibet, ou à la naissance du New Age », Études \\ mongoles et sibériennes, centrasiatiques et tibétaines [En ligne], 45 | 2014, mis en ligne le 30 juin 2014 \\ consulté le 13 juillet 2021. URL : http://journals.openedition.org/emscat/2423 ; DOI : https://doi.org/ \\ 10.4000/emscat.2423
}

Ce document a été généré automatiquement le 13 juillet 2021.

(c) Tous droits réservés 


\title{
L'exaltation des Roerich au Petit Tibet, ou à la naissance du New Age
}

\author{
Dany Savelli
}

Je remercie chaleureusement Quentin Devers, remarquable cartographe, pour l'élaboration de sa carte qui permet de visualiser l'expédition de Nicolas Roerich.

1 Le présent article n'entend pas revenir sur l'ensemble de l'expédition en Haute-Asie organisée par le peintre et mystique russe, Nicolas Roerich (1870-1947), entre mars 1925 et mai 1928. Son propos, plus modeste, est de s'attarder sur l'exaltation assez singulière qui s'empara du peintre, de sa femme, Elena (1879-1955), et de son fils aîné, Youri ${ }^{1}$, alors que leur caravane formée à Gulmarg traversait le Cachemire et le Ladakh pour rejoindre Khotan en Chine. L'intérêt pour nous sera de voir la façon dont cette exaltation conforta ces théosophes voyageurs à penser leur pérégrination à la fois comme une épopée et comme une ambassade destinées à faire basculer l'humanité dans l'Ėre nouvelle (Novaja Éraª qu'ils appelaient de leurs vœux. 


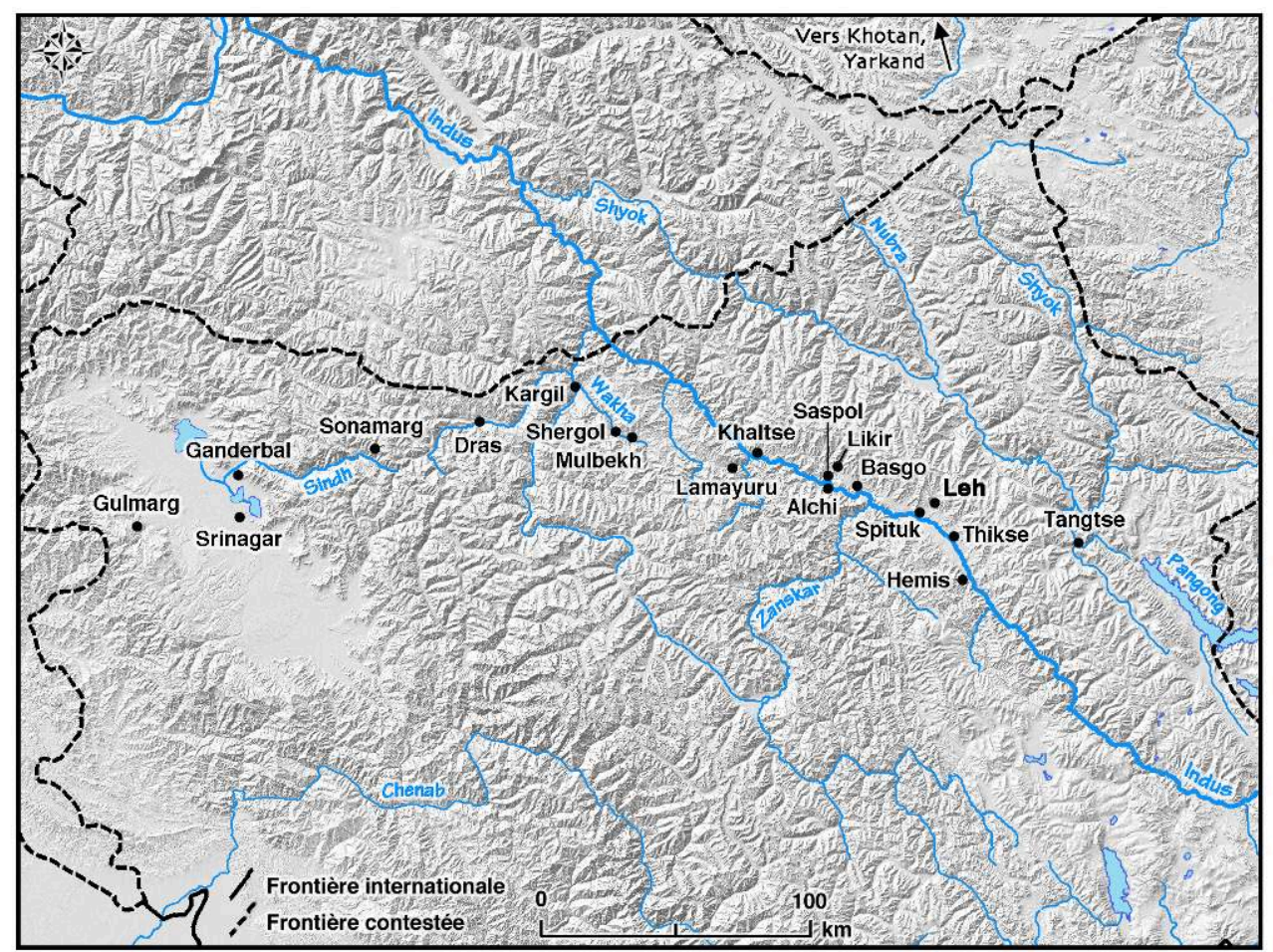

(c) Carte de Quentin Devers

Pour mieux nous aider à comprendre leur état d'esprit en mars et septembre 1925, quatre textes publiés à leur retour d'expédition seront considérés: d'une part, le journal de voyage de Youri, Trails to Inmost Asia, publié en 1931 dans lequel ce dernier rend compte avec précision de la topographie des zones traversées tout en insérant de longs développements sur les peuples, l'art et l'archéologie des lieux visités; d'autre part, Altai-Himalaya ; a Travel Diary publié en 1929 et Heart of Asia ${ }^{3}$ et Shambhala parus l'année suivante. Ces trois livres de Nicolas Roerich, traduits du russe par des proches, relèvent à la fois du récit viatique et de l'essai poétique. Composés de courts chapitres, eux-mêmes consistant en notations prises sur le vif, en impressions de voyages et anecdotes diverses, ils privilégient la forme brève et, ce faisant, autorisent un certain flou à même de transmettre l'enthousiasme du peintre en Asie. Le contraste entre les livres de Youri et ceux de son père permet de saisir comment leur exaltation, fondée sur des découvertes sensationnelles et le déchiffrement de signes prétendument prophétiques, fut contenue ou, au contraire, mise en exergue en fonction du lectorat à qui chacun s'adressait: averti, voire savant pour Youri, grand public et avide de merveilleux pour Nicolas.

3 Les découvertes incroyables du printemps et de l'été 1925 se produisirent à des points quasiment extrêmes du parcours considéré ici, à savoir Srinagar, la capitale du Cachemire, et Leh, la capitale du Petit Tibet comme on appelait alors le Ladakh. Dans la première de ces villes, les trois voyageurs eurent la surprise d'entendre raconter un épisode de la vie du Christ passé sous silence dans les Évangiles. Voici en quels termes Roerich rapporte cette légende :

The Moslems of Srinagar told us that the crucified Christ - or, as they call Him, Issa - did not die on the cross, but only lost consciousness. The disciples took away His body, secreted it and cured Him. Later, Issa was taken to Srinagar, where He taught 
the people. And there He died. The tomb of the Teacher is in the basement of a private house. It is said that an inscription exists there stating that the son of Joseph was buried there. Near the tomb, miraculous cures are said to take place and fragrant aromas to fill the air. In this way, the people of other religions desire to have Christ among them (1930, pp. 22-23).

Il n'est pas sans intérêt de noter dès à présent que les désignations du fils de Dieu sont ici en conformité avec l'enseignement théosophique qui, à la suite d'Édouard Schuré ([1889]-1997), considère le Christ comme un grand Initié. Il en va de même quand, rapportant deux autres versions de la vie du Christ entendues au Ladakh, Roerich qualifie de « grande sagesse » l'enseignement reçu par celui-ci :

In Leh, we again encountered the legend of Christ's visit to these parts. The Hindu postmaster of Leh, and several Ladaki Buddhists told us that in Leh not far from the bazaar, there still exists a pond, near which stood an old tree. Under this tree, Christ preached to the people, before his departure to Palestine. We also heard another legend of how Christ, when young, arrived in India with a merchant's caravan and how He continued to study the higher wisdom on the Himalayas. We heard several versions of this legend which has spread widely throughout Ladak, Sinkiang and Mongolia, but all versions agree on one point, that during the time of His absence, Christ was in India and Asia (1930, pp. 29-30).

5 Si au XVII e siècle, dans un mouvement somme toute naturel en regard des supputations sur le mystérieux Royaume du prêtre Jean, le premier Européen venu au Ladakh ${ }^{4}$ repéra avec enthousiasme un fond chrétien dans le bouddhisme tibétain, le cas de Roerich diffère considérablement : il ne s'agit pas pour lui de retrouver coûte que coûte des éléments chrétiens chez les peuples himalayens et de discréditer ainsi le bouddhisme en tant que christianisme dégénéré. Il s'agit de valider l'existence de cette " haute sagesse » (qu'on l'appelle philosophia perennis ou prisca theologia) à l'origine de toutes les traditions religieuses et que seuls, selon Mme Blavatsky, les membres de la Fraternité blanche, ou Mahatmas, retirés dans "les chaînes neigeuses des monts Himâlayas " (Blavatski 2004, p. 11) conservent intacte. Alors que Youri, avec précaution, se contente d'une rapide allusion aux «curieuses légendes de caractère chrétien » (1931 p. 29) répandues chez les musulmans cachemiriens et ladakhis, son père, lui, insiste sur les légendes de Srinagar et de Leh en vue d'accréditer la thèse de l'unité première des religions. Et comme si cela ne suffisait pas, il rapporte également la rumeur selon laquelle la treizième tribu d'Israël aurait autrefois trouvé refuge au Cachemire (1985, p. 212).

6 Peu importe que les « inventeurs » ou propagateurs de ces légendes soient connus; peu importe qu'il s'agisse, pour la mort du Christ à Srinagar et les origines juives des Cachemiriens, de Mirza Ghulam Ahmad (1835-1905), et, pour l'idée d'un Christ façonnant la doctrine bouddhique ou formé par elle, du Russe Alexandre Notovitch (1858- ?). Qu'importe même que le courant religieux fondé par Ghulam Ahmad ait été déclaré hérétique par les autorités de l'islam et que Notovitch ait de suite été démasqué comme un imposteur ${ }^{5}$, Roerich, à l'instar de Blavatsky, appartient «aux groupes de ceux qui sont convaincus qu'aucun récit mythologique, aucun événement traditionnel des légendes populaires, n'a jamais été, à aucune époque, une pure fiction, mais que chacun de ces récits possède un fond historique réel " (Blavatsky 2004, p.125). L'important n'est donc pas « de savoir où et comment la légende est apparue », mais de « noter qu'elle est racontée avec la plus totale sincérité » (N. Roerich 1930, p. 30).

7 Nicolas Roerich entend par ses livres témoigner de cette sincérité, comme il entend témoigner de la vigueur des légendes sur le Christ auprès des Cachemiriens et Ladakhis, 
toutes confessions et appartenances sociales confondues. Les musulmans, les bouddhistes, les hindous, et même, précise-t-il, la famille royale du Ladakh (N. Roerich 1985 p. 121) les répètent et, ce faisant, ajouterons-nous, leur donnent vie et valeur de vérité :

One might wonder what relation Moslems, Hindus or Buddhists have with Issa. But it is still more significant to see how vital are great ideas and how they penetrate even the most remote place (N. Roerich 1983, p. 89).

8 Cela dit, pas plus que son fils, Nicolas Roerich ne peut faire l'économie d'un argument solide à opposer aux incrédules : il s'agit des croix nestoriennes découvertes en 1906 par le missionnaire britannique F. E. Shawe à Tangtse, près du lac Pangkong à l'est de Leh et dont l'éminent spécialiste du Ladakh, August H. Francke (1870-1930), rendit compte pour une revue scientifique allemande en $1925^{6}$. Alors, comme l'écrit Youri, la présence «d'une population flottante de Chrétiens nestoriens au Ladakh entre les huitième et dixième siècles après J.-C. " pourrait bien expliquer que ces différents récits de la vie du Christ soient «des vestiges d'un passé nestorien» (1931, p. 28-29). Qui douterait de l'existence de ces légendes au Cachemire et au Ladakh, ajoute Roerich, ne comprendrait pas l'importance qu'eurent les Nestoriens «dans toutes les régions de l'Asie ».

9 Il nous semble que cette volonté de rattacher les légendes cachemiriennes et ladakhis à la présence intrigante de croix nestoriennes dans la région - thèse a priori intéressante et plausible - relève de la part de Nicolas et Youri Roerich du souci de se justifier. En effet, leur séjour au Cachemire et au Ladakh fit en 1926 l'objet d'une campagne de presse orchestrée par le Nicholas Roerich Museum dans le but évident d'assurer au peintre une visibilité d'important personnage public ; à cette occasion, plusieurs grands quotidiens américains lui avaient attribué la découverte d'un évangile apocryphe au monastère d'Hemis qui attestait la venue du Christ au Tibet ${ }^{7}$. Or, dans certains milieux, cette reprise du canular de Notovitch avait porté un préjudice considérable à la réputation des Roerich, et plus particulièrement à celle de Youri. On en donnera pour exemple la recension de son premier ouvrage, Tibetan Paintings, publiée par August Francke en 1927. Malgré l'intérêt de cette monographie, l'éminent savant de la Mission morave avouait s'être montré fortement indisposé à l'égard du jeune orientaliste en raison de ses affabulations ridicules sur un hypothétique évangile tibétain conservé à Hemis ; il expliquait également n'avoir accepté de recenser le livre de Youri que par égard pour le maître de ce dernier, le sinologue Paul Pelliot (1878-1945) ${ }^{8}$.

Sans prétendre à une carrière universitaire de type classique, Youri entendait bien s'affirmer comme un orientaliste et eût donc à défendre sa réputation. Une lettre de sa traductrice française, Marie de Vaux-Phalipau (ca.1862 - ap. 1946) en rapport avec le passage consacré aux croix nestoriennes dans son livre l'atteste ${ }^{9}$. De surcroît, Trails to Inmost Asia, ouvrage édité par des maisons d'édition à la réputation solide - les presses universitaires de Yale et, pour la traduction française, la Librairie Orientaliste Paul Geuthner -, visait à donner une caution scientifique à une expédition dont les organisateurs s'efforçaient d'occulter la dimension spirituelle. Mettre en avant leur credo de théosophes eut nui à la réputation de Nicolas Roerich alors que celui-ci entendait élaborer et faire reconnaître un pacte international portant son nom ${ }^{10}$. Pour parvenir à ses fins, la constitution d'un impressionnant réseau de personnes influentes et l'appui d'institutions respectables lui étaient nécessaires, dont celui du Vatican et de l'Église orthodoxe russe hors frontières guère disposés en faveur des doctrines fumeuses de Mme Blavatsky... On peut, sans risquer d'erreur, affirmer que Trails to 
Inmost Asia répondit à cette stratégie familiale, de même que le firent, à leur façon, les trois récits de voyage de Nicolas Roerich.

11 Lorsque dans Altai-Himalaya, ce dernier revient sur l'hostilité des missionnaires ${ }^{11}$, dont, à n'en pas douter, celle de Francke :

Regarding the legends of Jesus - first there was a complete denial. To our amazement denial first comes from the circle of missionaries. Then slowly, little by little, creep in fragmentary, reticent details, difficult to obtain. Finally it appears that the old people in Ladakh have heard and know about the legends (1983, p. 114)

on notera qu'il présente ces légendes sensationnelles sur le Christ comme si les Frères moraves, installés au Ladakh depuis la fin du XIX siècle, devaient s'en réjouir à la façon des Jésuites portugais $d u \mathrm{XVII}^{\mathrm{e}}$ siècle convaincus de retrouver des éléments chrétiens chez les « lamaïstes » du Tibet. Or l'époque avait changé et Roerich était assez fin pour le savoir ${ }^{12}$. On peut, nous semble-t-il, déceler là un subterfuge de sa part, tout comme peut-être son choix de faire des croix nestoriennes un argument pour accréditer la thèse d'une origine commune au bouddhisme et au christianisme : après tout, Francke, on l'a vu, avait été un des premiers, sinon le premier à écrire à leur sujet.

13 Cependant, toutes ces légendes qui bouleversaient les enseignements chrétiens n'expliquent pas à elles seules l'exaltation qui gagna les Roerich sur l'ancienne piste des caravanes menant à Leh. Car si elles renforcèrent leur credo de théosophes, elles produisirent vraisemblablement un effet moindre sur eux que certains bas-reliefs aperçus sur le bord de la route et plusieurs statues immenses encloses dans l'espace étroit des temples. Ces œuvres auxquelles nous faisons ici allusion comblèrent leur attente millénariste : toutes représentaient Maitreya, le Bouddha du Futur, destiné à établir la paix universelle.

14 Ce fut d'abord, près de Dras, un bas-relief représentant le Bodhisattva debout en " costume princier, un rosaire dans la main droite, un vase ou bum-pa dans la gauche » (Y. Roerich 1931, p.10). Pour Roerich, le caractère remarquable de ce "premier message bouddhique » fut qu'il représentait "précisément la figure de Maitreya » (1983, p. 102). Le second "emblème bouddhique» rencontré en cheminant fut le somptueux Maitreya du viII ${ }^{\mathrm{e}}$ siècle dans le village de Mulbekh. Gravée dans un piton rocheux qui, à cet endroit, force la route à marquer un tournant (et les voyageurs à l'admirer, voire l'adorer...), la divinité à quatre bras, parée de ses bijoux et coiffée d'une tiare surmontée d'un petit stupa, ne manqua pas par sa grâce d' "ascète indien " associée à son caractère monumental de susciter "une impression inoubliable et puissante » (Y. Roerich 1931, pp. 13-14) même si, à cette époque déjà, un petit temple en masquait en partie la vue. 


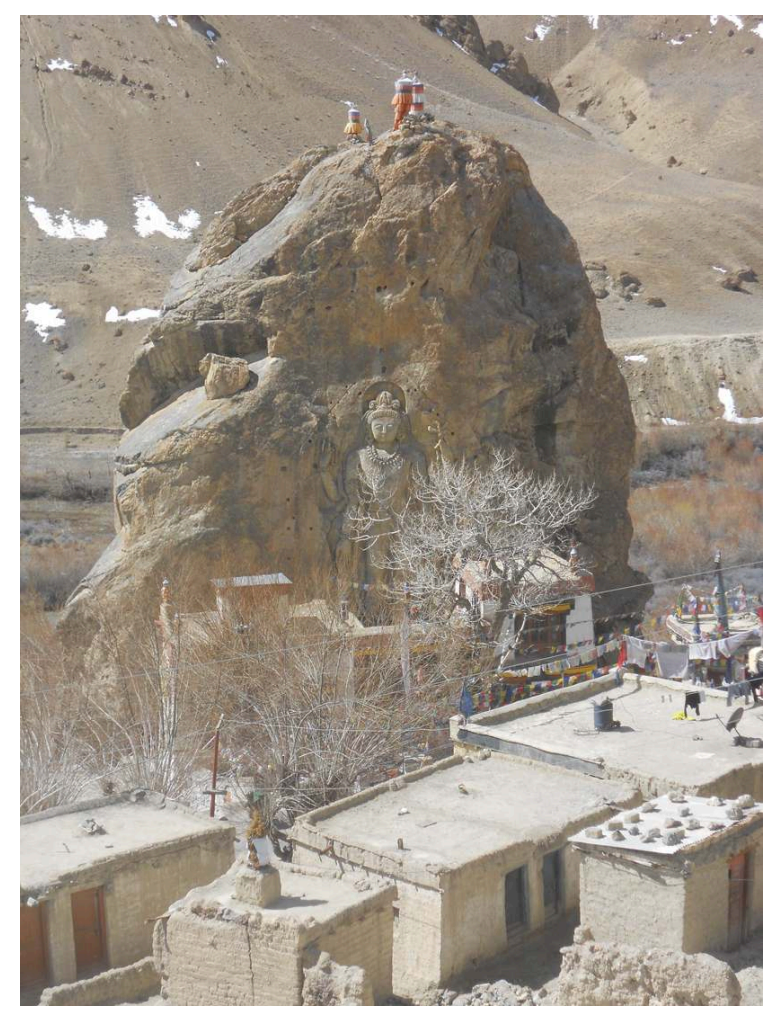

Photo de Dany Savelli (avril 2014)

Puis de cette figure en majesté du Bodhisattva jusqu'à Thikse à l'est de Leh, la route formait une guirlande de temples dont il suffisait de pousser les lourdes portes en bois pour aussitôt faire face à d'immenses Bouddha du futur. C'étaient les Maitreya du temple de Sumtsek à Alchi, du monastère de Saspol, des temples de Cham Chung, Serzang et Chamba Lhakhang ${ }^{13}$ du monastère de Basgo, du Chamba Lhakhang du Palais royal de Leh, du Tsemo Gompa qui surplombe ce palais, et enfin du temple Chamkhang au monastère de Thikse. À cette série de Boudhha du Futur dûment mentionnés par les Roerich, on ajoutera la peinture murale d'un Maitreya au monastère de Spituk à laquelle il est fait allusion dans Altaï-Himalaya (pp. 112-113).

Comment expliquer cette insistance sur l'omniprésence de Maitreya au Ladakh alors que Nicolas et Youri taisent l'existence de statues tout aussi monumentales des bodhisattvas Avalokiteshvara et Manjushri? Par la grande dévotion dont Maitreya jouit encore aujourd'hui au Ladakh ${ }^{14}$ ? Ou par la volonté de mettre en exergue une figure messianique réconciliant deux grands courants religieux? Maitreya ne fait-il pas l'objet de dévotion aussi bien dans l'école Théravada que dans l'école Mahayana? N'est-il pas, pour citer Altaï-Himalaya (p. 104), "le seul à unir fermement le Hinayana et le Mahayana y compris à Ceylan»? Ces faits sont bien sûr à retenir, cependant, n'oublions pas que les Roerich appartenaient à cette seconde génération de théosophes - appelée parfois néo-théosophes - qui, sous l'impulsion de Charles Leadbeater (1854-1924) et d'Annie Besant (1847-1933), identifièrent Maitreya au Christ et annoncèrent l'imminence de son avènement. Quelle que fût la hargne d'Elena à dénigrer tous ses contemporains qui prétendaient, comme elle, détenir leur enseignement directement des Maîtres de Blavatsky, les Roerich n'en partagèrent pas 
moins avec la plupart d'entre eux un millénarisme qu'on ne trouve pas chez l'auteur de la Doctrine secrète.

17 Vivant dans l'attente impatiente de l'établissement d'un royaume de justice, de la venue d'un libérateur, de l'avènement de temps nouveaux et de l'émergence d'un Nouveau Pays (Novaja Strana) ${ }^{15}$, les Roerich étaient avides de présages et de signes. Or, dans ce Petit Tibet si proche du légendaire Shambhala, ces signes abondaient, du moins eux les voyaient-ils, les entendaient-ils. "Toute l'atmosphère du Ladakh semblait chargée d'éléments positifs pour nous ", reconnut plus tard Roerich (1930, p. 30). Il suffisait de relier les légendes, les mythes et les prophéties pour déchiffrer présages et signes. À la faveur de certaines circonstances, leur multitude et leur vigueur pouvaient susciter de véritables hallucinations. Ainsi lorsque l'artiste à l'origine du Sacre du Printemps ${ }^{16}$ plaçait sur son gramophone portatif un enregistrement sur disque de Wagner, pouvait-il voir projeté sur les parois des montagnes himalayennes l'embrasement apocalyptique attendu et toute la nature se métamorphoser en une scène sublime narrant une épopée grandiose. Ce qui avait été conçu en Occident de façon illusoire pour les besoins des scènes d'opéra prenait vie au Petit Tibet. Un étonnant passage d'Altai-Himalaya le raconte :

Nature, having no outlet, inscribed epics with their wealth of ornamentation, on the rocks. One perceives how the forms of imagery blend with the mountain atmosphere. Just those forms, thought out in the West, here begin to live and become convincing. One may expect the appearance of Kuan Yin; or Lhamo prepares the element of destruction; or the image of Mahakala may issue from the mass of destruction. This is not the unlifelike (sic) Durandale from Rockamadura. And how many enchanted helmets and swords are hidden in the chasms! This is the real tragedy and achievement of life. And Bruguma of Gessar Khan is kin to Brunhilde of Siegfried. Crafty Locke runs along the fiery rocks (1983, p. 107) ${ }^{17}$.

À cette description hallucinée servie par la musique de Wagner - «elle résonne remarquablement dans les montagnes » (N. Roerich 1983, p. 107) - s'ajoute le compterendu des rumeurs, qui, tels les messages d'une conspiration, circulent à mots couverts comme empreintes de terreur sacrée. En effet, alors que la caravane progresse avec lenteur, que l'air raréfié ajoute à la fatigue et que «les jours et les dates sont rapidement oubliés" (N. Roerich 1983, p.83), le pays ladakhi, plongé dans une temporalité en suspens, bruisse de l'avènement de l'Ėre nouvelle : en ces lieux reculés, les lamas s'entretiennent de Maitreya en chuchotant (N. Roerich 1983, p. 104).

On croirait à tort cependant que la magnificence des paysages cachemiriens et ladakhi et l'omniprésence de Maitreya furent la seule cause de l'exaltation des Roerich. En réalité, ils entrèrent au Cachemire et au Ladakh déjà amplement confortés dans leur conviction de l'imminence de l'Ėre Nouvelle et ce, en raison de trois événements survenus un peu plus tôt. D'abord, en 1924, au Sikkim, la nouvelle de la fuite du Panchen Lama leur était parvenue : la prophétie tibétaine selon laquelle le départ du Tibet de ce hiérarque marquerait la venue des guerriers de Shambhala était donc enclenchée. Ensuite, le 25 octobre 1924 à Calcutta, Alexandra David-Néel (1868-1969) révélait à Roerich que les Tibétains vivaient dans l'attente imminente d'un "saint guerrier qui régénérera[it] le monde » et que Shambhala, assimilé au royaume de Gesar, le héros de l'épopée tibétaine, se situait quelque part au nord dans une contrée peuplée de Russes ${ }^{18}$. Il n'en fallait pas davantage pour que les Roerich, qui jusque-là n'avaient jamais entendu parler de Gesar mais connaissaient le mythe eschatologique de Shambhala, associent messianisme tibétain et révolution bolchevique. Ils se trouvaient 
d'autant plus confortés à le faire que durant les années 1920, le Kremlin encourageait une politique de concessions visant, entre autres, à attirer de grandes firmes étrangères pour contribuer au développement de régions éloignées du pays; en 1924, le gouvernement soviétique créait même à cet effet un bureau à New York ${ }^{19}$. Les Roerich s'engouffrèrent dans cette brèche. À la fin de l'année, au cours d'un voyage éclair à New York, Paris et Berlin, Nicolas collectait des fonds auprès de plusieurs millionnaires américains, créait la société Beluxa et approchait des agents et des diplomates soviétiques pour discuter de sa venue à Moscou et obtenir une concession dans l'Altaï ${ }^{20}$. Le journal d'Elena $(2011,2012)$ ne laisse cependant aucun doute à ce sujet : les Roerich envisageaient bien cette concession comme une utopie bouddho-communiste et l'Altaï, nouveau siège des Mahatmas, comme un substitut soviétique du Tibet. Compte tenu de cet état d'esprit, les signes et présages qu'ils crurent reconnaitre au Ladakh, avant que leur utopie ne se fracassât sur la dure réalité soviétique, furent une composante émotionnelle déterminante de l'aventure au cours de laquelle Nicolas Roerich finit par s'identifier à une figure messianique destinée à instaurer l'Ėre nouvelle.

\section{BIBLIOGRAPHIE}

Archer, K.

1990 « Nicolas Roerich et la genèse du Sacre », in Le Sacre du printemps de Nijinsky (Paris, Cicero), pp. 75-95.

Banerjee, Partha S.

2010 [2008] Ladakh. The Essential Guide with Kashmir and Kulu-Manali (Calcutta, Milestone Books).

Blavatsky, H. P.

2004 Abrégé de la doctrine secrète (Paris, Éditions Adyar).

David-Néel, A.

1925 Prophéties et légendes tibétaines : le futur héros du Nord, La Vie des peuples, 61, pp. 156-161.

Didier, H. (éd.)

1996 Les Portugais au Tibet. Les premières relations jésuites (1624-1635) (Paris, Éditions Chandeigne).

Francke, A. H.

1927 « Roerich, George, Tibetan Paintings, Paris, Paul Geuthner, 1925 », Orientalistische

Literaturzeitung, col. 408-410.

Notovitch, N.

2004 [1894], La Vie inconnue de Jésus-Christ, préf. de Christian Bouchet (Paris, Pardès).

Rerih [Roerich], E.

2009 Listy dnevnika. 1920-1923, éd. de Vladimir Rosov (Moscou, Russanta - Gosudarstvennyj Muzej

Vostoka).

2011 Listy dnevnika. 1924-1925, éd. de V. Rosov (Moscou, Russanta - Gosudarstvennyj Muzej

Vostoka).

2012 Listy dnevnika. 1925-1927, éd. de V. Rosov (Moscou, Russanta - Gosudarstvennyj Muzej

Vostoka). 
Roerich, G. [Youri]

1931 Trails to Inmost Asia Trails to Inmost Asia. Five Years of Exploration With the Roerich Central Asian Expedition, préf. de Louis Marin (New Haven, Yale University Press ; Londres, H. Milford - Oxford University Press).

Roerich, G. de

1933 Sur les Pistes de l'Asie centrale, préf. de Louis Marin, trad. de Mme de Vaux-Phalippau (Paris, Librairie Orientaliste Paul Geuthner).

Roerich, $\mathrm{N}$.

1983 [1929] Altai-Himalaya; a Travel Diary, introduction de C. Bragdon (New York, Frederick A.

Stokes Company).

1930 Heart of Asia (New York, Roerich Museum Press).

1985 [1930] Shambhala (New York, Frederick A. Stokes company).

Rerih [Roerich], N.

1929 Serdce Azii (Southbury, Alatas).

Rosov, V.

2002 Nikolaj Rerih. Vestnik Zvenigoroda. Èkspedicii N.K. Reriha po okrainam pustyni Gobi, t. I, (Saint-

Pétersbourg, Aletejja SPB - Ariavarta-Press).

2005 Amerikanskoe obščestvo "Beluha” i proekt N.K. Reriha "Edinaja Azija”, Vestnik Sankt-

Peterburskogo universiteta, vyp. 2, pp. 65-75.

Savelli, D.

2011 L'expédition Roerich (1925-1928) en quête de Shambhala d'après les coupures de presse du Nicholas Roerich Museum, in D. Aigle, I. Charleux, V. Goossaert \& R. Hamayon (éd.), Festschrift in Honour of Françoise Aubin, Institut Monumenta Serica, Sankt Augustin, pp. 781-811.

2013 Alexandra David-Néel et Nicolas Roerich : histoire d'une rencontre autour de Gesar de Ling et de Shambhala, Politica Hermetica, 27, pp. 150-167.

[en relecture] Trails to Inmost Asia (1931) de Youri Roerich : une caution scientifique à l'Expédition Roerich en Asie centrale (1925-1928) ?, Journal asiatique.

Schuré, É.

1997 [1889] Les Grands Initiés. Rama - Krishna - Hermès - Moïse - Orphée - Pythagore - Platon - Jésus

(Paris, Perrin).

Sims-Williams, N.

1993 The Sogdian inscriptions of Ladakh in Karl Jettmar, Ditte König \& Martin Bemman (éd.), Antiquities of Northern Pakistan (Mainz, P. von Zabern), pp. 151-163.

Špotov, B. M.

2013 Amerikanskij biznes i Sovetskij Sojuz v 1920-1930-e gody. Labirinty èkonomičeskogo sotrudničestva (Moscou, urss).

\section{NOTES}

1. Youri Roerich (Jurij Rerih) (1902-1960) signa ses publications en anglais en utilisant le prénom George, écrit Georges pour ses publications en français. Pour désigner l'auteur de Trails to Inmost Asia, nous utiliserons dans cet article son prénom russe, Youri. Le nom de famille Roerich, sans mention de prénom, sera réservé à son père, Nicolas. Notons qu'après avoir obtenu, par faveur spéciale, un passeport français en 1930, mais non la nationalité française, Nicolas et Youri Roerich firent souvent précéder leur nom de famille de la particule « de ». 
2. La promesse d'une «Ére nouvelle » apparaît pour la première fois dans le journal d'Elena Roerich le 27 septembre 1922 (E. Rerih 2004, p. 444). Rappelons que ce journal correspond à la retranscription scrupuleuse des messages qu'à partir de 1920 et ce jusqu'à sa mort, elle reçut chaque jour ou presque de Morya, ce même Maître de sagesse doté de pouvoirs surnaturels avec qui Mme Blavatsky (1831-1891) prétendait s'entretenir.

3. Dans cet article, je ne prendrai pas en compte la première version de ce livre (Rerih 1929). Publié en russe par une petite maison d'édition subventionnée par le Nicholas Roerich Museum (lui-même fondé à New York en 1923 et regroupant diverses institutions), cet ouvrage s'adressait à un lectorat restreint et sensiblement différent du grand public anglo-saxon que Roerich souhaitait atteindre avec Heart of Asia. Je réserve pour une étude plus développée une comparaison des deux versions.

4. En 1603, le marchand Diogo de Almeida décrit les temples de la ville de Basgo, une des anciennes capitales du Ladakh située sur la rive droite de l'Indus, comme des " églises richement ornées de retables et d'images de Notre Seigneur Jésus-Christ, de Notre Dame et des saints Apôtres ». Pour cette citation, $c f$. Didier 1996, p. 293.

5. Au sujet d'Alexandre Notovitch et Mirza Ghulam Ahmad, $c f$. la préface de Christian Bouchet in Notovitch 2004, pp. 7-30.

6. À ce sujet, de même que pour une remise en question du caractère chrétien de plusieurs de ces croix, $c f$. Sims-Williams 1993, p.151. Je remercie Laurianne Bruneau pour m'avoir signalé cet article.

7. Cf. Savelli 2011. Dans la mesure où le présent article s'inscrit dans la préparation d'une monographie consacrée à l'Expédition Roerich en Haute-Asie, nous n'aurons d'autre choix, pour éviter au mieux les redites, que de renvoyer à plusieurs reprises à nos publications antérieures.

8. De toute évidence, Francke confondit Youri et son père, car la découverte de l'évangile d'Hemis fut attribuée par la presse à Nicolas Roerich et non à son fils. Pour le préjudice que cette affaire causa à Youri dans les milieux orientalistes, français notamment, cf. Savelli (en relecture).

9. Cf. Ibid.

10. Ce pacte, destiné à préserver les œuvres d'art en temps de guerre, fut signé à Washington en 1935 par les États-Unis et vingt pays d'Amérique latine.

11. Il le fait encore dans Shambhala (1985, p. 125) en se moquant avec ironie des missionnaires protestants qui l'interdisent de prosélytisme au Ladakh. L'historien Vladimir Rosov (2002 p. 135) révèle qu'à Spiti comme au Ladakh, les Roerich répandirent la nouvelle de la venue de Maitreya et, avec l'aide de lamas, distribuèrent des pamphlets à ce sujet. Il serait bien entendu passionnant de savoir s'ils remportèrent du succès et si, dans le même temps, ils se livrèrent à de la propagande communiste.

12. Roerich connaissait d'ailleurs les écrits des religieux portugais venus au Tibet ; ils avaient été édités par Cornelius Wessels (1880-1964) à la Haye en 1924. Cf. Roerich 1930, pp. 160-161.

13. Chamba est le nom tibétain de Maitreya. Lhakhang désigne un temple.

14. Signe de cette dévotion, en 1980, la statue que virent les Roerich à Thikse a été remplacée par une nouvelle statue plus grande encore, en argile et métal dorés. CEuvre de l'artiste Nawang Tsering, elle passe pour la plus belle statue du Ladakh. Cf. Banerjee 2010, p. 158. On mentionnera également au monastère de Likir, à l'ouest de Leh, une statue d'un Maitreya assis. Haute de 23 mètres, elle a été achevée en 1999 et placée à l'extérieur des temples. Entièrement dorée, elle est particulièrement impressionnante quand de la vallée de l'Indus on approche le monastère construit sur une petite hauteur.

15. L'expression Nouveau Pays, qui se décline en Nouveau Monde (Novyj Mir) ou Nouvelle Russie (Novaja Rossija), est récurrente dans le journal d'Elena Roerich. Notre thèse, que nous ne pouvons ici développer, est que le parcours spirituel des Roerich fut largement dicté par la situation d'émigrés et d'apatrides qui fut la leur à partir de 1917. Le Nouveau Pays qu'ils évoquent est une utopie certes, mais c'est aussi le pays perdu à retrouver et à reconquérir. Le vocabulaire guerrier 
employé dans le journal d'Elena atteste que le retour à la terre natale est pensé comme une épopée au cours de laquelle des batailles seront à livrer, fussent-elles toutes diplomatiques. L'expérience de l'émigration nous semble distinguer les Roerich des épigones de Mme Blavatsky et de Blavatsky elle-même qui, née russe, prit la nationalité américaine en 1878.

16. Roerich est l'auteur de nombreuses affabulations sur sa biographie, néanmoins il est à présent avéré que la paternité de l'argument du Sacre du printemps (1913) ne revient pas à Stravinski, comme ce dernier le prétendit, mais bien à Roerich, signataire des décors et costumes du ballet et, en outre, connaisseur avéré des rites païens de l'ancienne Russie. Cf. Archer 1990, pp. 75-95.

17. Kuan Yin (Guanyin) : boddhisattva de la compassion. Palden Lhamo : déité courroucée, femme de Mahakala.

18. Pour la citation, $c f$. David-Néel 1925, p. 157. Sur les détails de la rencontre entre l'exploratrice française et le peintre russe et le fait que bien plus tard, celle-ci comprit que le héros légendaire évoqué par les rumeurs n'était autre que Roman von Ungern-Sternberg (1885-1921), libérateur de la Mongolie en février 1921, cf. Savelli 2013.

19. À ce sujet, $c f$. Špotov 2013.

20. Sur ces tractations, $c f$. Rosov 2005.

\section{AUTEUR}

\section{DANY SAVELLI}

Dany Savelli est maître de conférences à l'Université Toulouse Jean Jaurès et directrice de publication de la revue Slavica Occitania. Elle travaille sur l'imaginaire de l'Asie (Mongolie, Chine, Japon, Tibet) dans la pensée et la littérature russes, de même que sur le bouddhisme en Russie. Elle prépare actuellement une monographie sur l'Expédition en Asie centrale de

N. K. Roerich (1925-1928). 